\title{
Heart visualization based on hybrid transfer function using size and gradient
}

\author{
Yong Xia*, Yixuan Liu and Kuanquan Wang \\ Department of Computer Science and Technology, Harbin Institute of Technology, Harbin, China
}

\begin{abstract}
Having the ability to visualize the heart clearly and precisely would be beneficial for pathology research, presurgical planning, and clinical approaches. Multi-dimensional transfer functions were employed to improve the overall performance of images. To provide a satisfactory visualization quality on the shape and boundaries of the heart, a new hybrid transfer function combining structure size with gradient was designed to highlight the area of the heart. Initially, a histogram of gradient and histogram of size was computed and then classification was performed for providing the spatial information. Finally, several hybrid strategies were presented for the design of the transfer function, including opacity and color. By experimental evaluation, the proposed hybrid transfer function visualized the cardiac outline and internal structure more clearly and easily.
\end{abstract}

Keywords: visualization of heart, multi-dimensional transfer function, histogram of structure size, histogram of gradient

\section{Introduction}

The heart is one of the most important organs in the human body. Visualization of the anatomy of the human heart and a representation of its spatial characteristics in the chest cavity would be beneficial for pathology research, presurgical planning, and clinical approaches. Volume rendering methods have been widely applied in visualization, and the transfer function is a key process for volume rendering.

Several early designs for transfer function methods were discussed by Hanspeter Pfister et al. [1]. Initially, a one-dimensional transfer function was used with the opacity of voxels defined by the scalar value itself, which meant that voxels shared the same opacity with voxels of similar scalar value. In order to classify the voxels more accurately, a multi-dimensional transfer function was proposed in which opacity values were defined not only based on scalar value, but also other information, such as gradient value [2]. Levoy used gradient magnitude to design a transfer function [3]. By using the gradient magnitude, organ boundaries could be emphasized by assigning them a higher opacity than the interior parts.

However, ambiguity exists for gradient histograms when two arches overlap in boundary voxels, which can cause voxels to be classified incorrectly. Roettger et al. introduced a method to classify bins in gradient histograms [4]. In this new method, each bin contained scalar value and gradient

\footnotetext{
*Corresponding author: Yong Xia, Harbin Institute of Technology, P.O.box 332, 92 West Da Zhi St., Harbin, P.C.150001, China. Tel.: +86535 5687921; Fax: +86535 5687627; E-mail: xiayong@hit.edu.cn.
} 
magnitude, and also contained spatial information, like the barycenter or variance of each voxel in the same bin.

Structure size is important information extracted from original datasets to generate transfer functions. Runzhen et al. introduced a way to obtain size information based on region growing where several seeds were randomly selected for regional growth [5]. Correa and Ma employed a scale space method for assigning size information to each voxel [6]. However, in that method, the size information and scalar values were independent to establish two one-dimensional histograms for transfer functions. In this paper's proposed method, each voxel size is calculated based on a tolerant value set by the user, and a histogram is generated using size information and intensity. In addition to structure size, occlusion spectrum can also be a second feature employed to design the transfer function [7].

This study provided a method for visualization of the heart, as described in Section 2, including the visualization pipeline, feature extraction, histogram of feature and classification, and the hybrid transfer function. Then, experimental results and the conclusion are given in Sections 3 and 4, respectively.

\section{Method}

\subsection{Overview of visualization pipeline}

The visualization pipeline provides a mechanism to encapsulate algorithms and then couple them together in a variety of ways. Most prevalent visualization pipelines were proposed by Kenneth Moreland [8]. In this paper, the volume rendering process was divided into four parts, as shown in Figure 1. Initially, the feature values were extracted from the original dataset, which was used to distinguish different tissues. Then, histograms were generated based on intensity, gradient, and size features and classified by spatial information. According to the information provided by the histograms, the parameters for the design of opacity and color semi-autonomously were adjusted. Finally, direct volume rendering was adopted for the visualization of the 3D heart dataset.

\subsection{Feature extraction}

In image datasets obtained from CT and MRI equipment, noise is inevitable, and can affect the results of visualization because the calculation of the structure size is based on homogeneity assumptions. In this paper, a method of nonlinear diffusion filtering was adopted to reduce noise while retaining local details and boundary information [9].

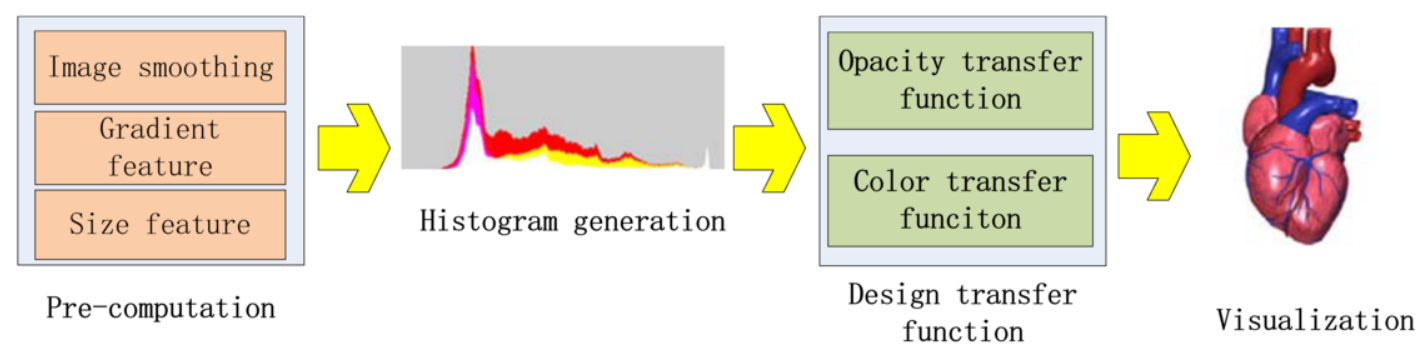

Fig. 1. Overview of visualization pipeline. 
Gradient is a very effective and popular feature for transfer function, and was also used in this paper. Additionally, a more promising feature of structure size was performed for cardiac visualization. The method of extracting size features is described as follows.

In three-dimensional volume data, there are 26 neighbors around each voxel. Therefore, 26 directions can be defined from the center voxel to each of its neighbors. Then, the size of a reference voxel can be obtained by voxel growth based on a similarity evaluation of its neighbors. The stop criterion for growth is the intensity variation within a special threshold as:

$$
\tau_{\text {range }}=\left[I_{\text {ref }}-\tau \cdot I_{\max }, I_{\text {ref }}+\tau \cdot I_{\max }\right]
$$

where $I_{\max }$ is the maximum intensity of all voxels in the data set, $\tau$ is a tolerance value in the interval $(0,0.5)$ and $I_{\text {ref }}$ is the gray value of the reference voxel. For each reference voxel, Eq. (1) can be used to acquire a size. The number of voxels in a size is a size feature value for the reference voxel, which will be adopted for the design of the opacity transfer function in Section 2.4.

\subsection{Histogram of feature and classification}

Histograms are commonly used to present statistical properties [10]. Histograms of intensity-gradient (HIG) and histograms of intensity-size (HIS) are also used. In HIS, the x-axis is the intensity and $y$-axis is the size feature, and this coordinate is defined as one bin. However, traditional histograms only show statistical information of voxels, while ignoring the spatial characteristics. This means that voxels in two adjacent bins in the histogram may be located anywhere in the 3D dataset. In order to solve this problem, a method was used similar to the method developed by Roettger et al. [4], where a histogram with spatial information was enhanced by computing the barycenter $b(T)$ and the variance $v(T)$ of all voxels that fall into the same $2 \mathrm{D}$ bin $T$.

Let $p_{i}(g, s)=\left(x_{i}, y_{i}, z_{i}\right), i=1 \ldots n$ be the normalized positions of contributing voxels in the same bin whose intensity is $\mathrm{g}$ and extracted size feature value is $\mathrm{s}$.

Let $b(g, s)=\frac{1}{n} \sum_{i=1}^{n} p_{i}(g, s)$ be the barycenter of voxels in the same bin, and let $v(g, s)=\frac{1}{n} \sum_{i=1}^{n}\left\|p_{i}(g, s)-b(g, s)\right\|$ be the spatial variance of voxels in the same bin.

For a referenced bin $T_{o}=(s, t)$ and arbitrary $T$, the distance norm between them can be expressed by the following equation:

$$
N\left(T, T_{o}\right)=\left\|b(T)-b\left(T_{o}\right)\right\|+\left|v(T)-v\left(T_{o}\right)\right|
$$

And if $\forall T, N\left(T, T_{O}\right)<r$, then $T_{O}$ and $T$ belong to the same category. For classifying all bins, this paper started from bin $T_{O}$, which contained the largest number of voxels as a reference bin. Then, the unclassified bins were traversed to determine whether they belonged to the same class, and lastly, 
each class was given a unique color. This procedure was repeated for the remaining entries until the classification was completed. By repeating this operation, it was discovered that histograms not only contain statistical information, but also include spatial characteristics. The results of the classification were adopted for the design of the color transfer function described in Section 2.4.

\subsection{Hybrid transfer function based on size and gradient}

A hybrid transfer function combining HIS and HIG was developed. In addition, feature classification was adopted for color design and direct volume rendering was used for the best performance. Figure 2 provides the system diagram for the hybrid transfer function. The design of the transfer function included opacity and color.

\subsubsection{Opacity}

The opacity should increase with the decrease of the size value or the increase of the gradient value so that the boundaries can be highlighted. The opacity of a voxel is defined as $\alpha$, and the values in HIS and HIG are defined as s and g, respectively. The size feature value, that is $\mathrm{s}$, can be obtained according to the method in Section 2.2.

Threshold, sigmoidal, and power-law hybrid strategy were used for opacity design as Eqs. (3)-(5), respectively, which are similar to [11].

$$
\begin{aligned}
& \alpha=o(T, w, g, s)=\left\{\begin{array}{l}
0 \quad, w \times g+(1-w) \times s>T \\
w \times g+(1-w) \times s, w \times g+(1-w) \times s>T
\end{array}\right. \\
& \alpha=o(T, r, w, g, s)=\frac{1}{1+e^{-r[w \times g+(1-w) \times s-T]}} \\
& \alpha=o(k, r, w, g, s)=k \times[w \times g+(1-w) \times s]^{r}
\end{aligned}
$$

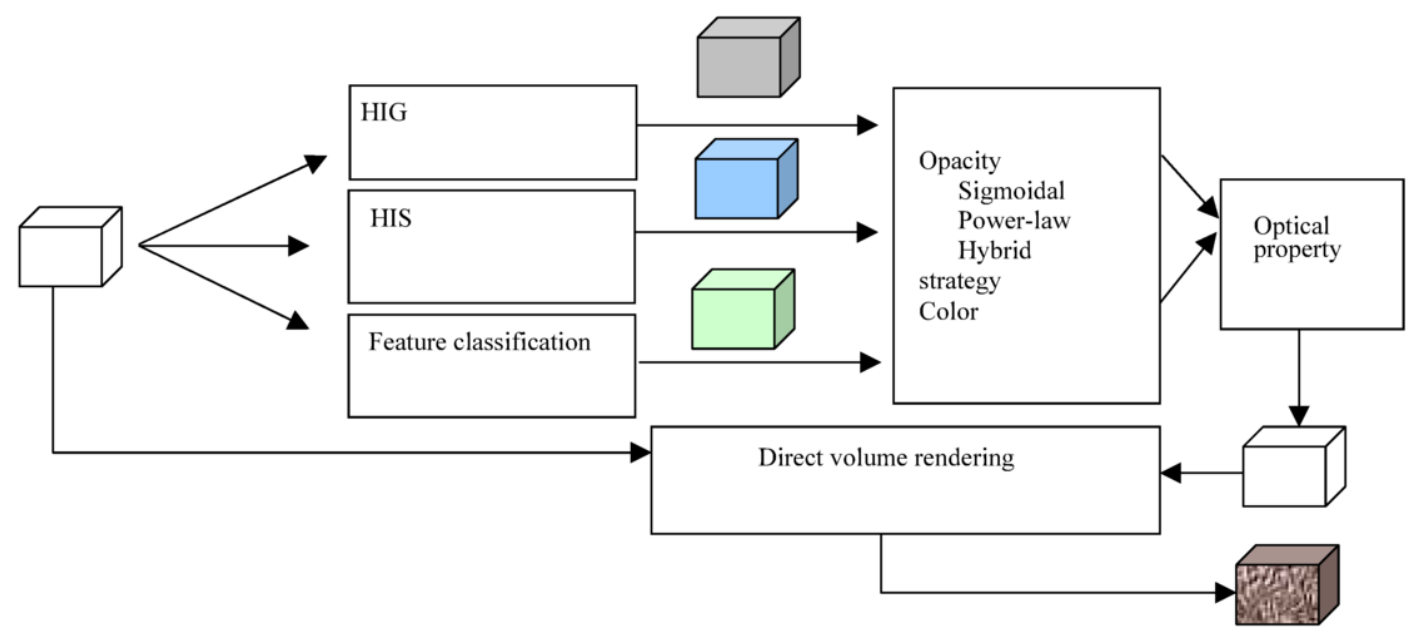

Fig. 2. System diagram for the design of hybrid transfer function. 
where $\mathrm{T}$ is a threshold, $\mathrm{w}$ is a weighting factor, $\mathrm{r}$ is a control parameter for transition period, and $\mathrm{k}$ is a ratio.

\subsubsection{Color}

For color design, the feature classification described in Section 2.3 was adopted. All voxels in a class were assigned the same color. In this paper, a HSV model was used for color design, because it is an intuitive color model for the human body. Assume in a dataset, the maximal size value is Max, and the minimal size value is Min. The scope for Hue $(\mathrm{H})$ is 0 to 360 . As such, the value of Hue by linear mapping can be obtained from Max to 360 and from Min to 0. For better performance, logarithmic mapping was used. Saturation (S) and value (V) in HSV depended on the size and gradient information. With increasing gradient and decreasing size, saturation decreased linearly, while value linearly increased. This caused the darkening of the boundaries as well as color attenuation for larger structures. In this paper, these two parameters were set by the user based on interactive operation.

\section{Result}

The data of a sheep's heart, with a size of $352 \times 352 \times 240$, was utilized for the visualization evaluation. The results are given in Figures 3 and 4. Figure 3 shows the results based on HIG only, and the colors were defined by the number of voxels in each group. Bins of high gradient value are recognized by a yellow arch, which meant a boundary in the original dataset, then by definition of the opacity transfer function that assigned high opacity values to high gradient values, the boundaries are clearly visualized by the yellow color in the right image. Figure 4 shows the results based on both HIG and HIS using Eq. (5), by adjusting parameter $\mathrm{w}$ to balance the size and gradient features. High opacity was assigned to voxels with high gradient values and small size values to highlight the boundaries.

Figure 5 shows an experiment for viewing homogeneous regions. The left picture is the original dataset of the sheep heart and the rectangular region is a homogeneous region whose gray values are similar. This region can be easily picked up in HIS and the boundaries are expressed clearly in HIG. Users can select the blue region in HIS by mouse as the gray rectangle, as shown in Figure 5, and then the corresponding voxels in the original dataset can be visualized by Eq. (5), as the picture on the right shows in Figure 5. Using the hybrid transfer function, the red structure was visualized by size information and other structures were based on gradient magnitude by adjusting parameter $\mathrm{w}$. By comparing the results of $\mathrm{A}$ and $\mathrm{B}$, it can be seen that the region was not visualized completely by HIG alone, and some voxels were lost.

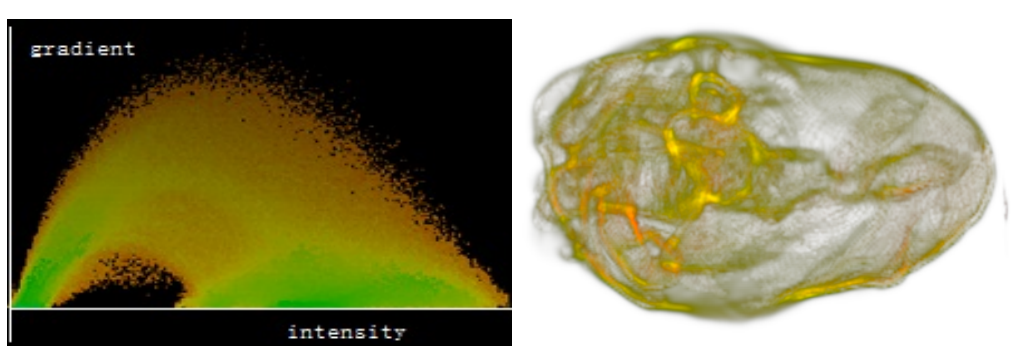

Fig. 3. Visualization of heart based on HIG. 

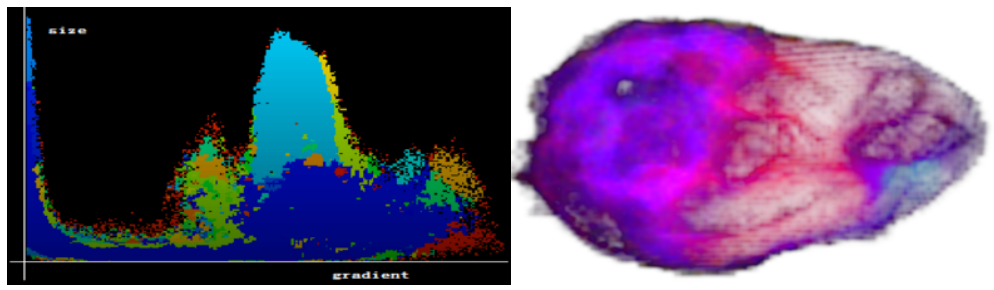

Fig. 4. Visualization of heart based on hybrid transfer function.

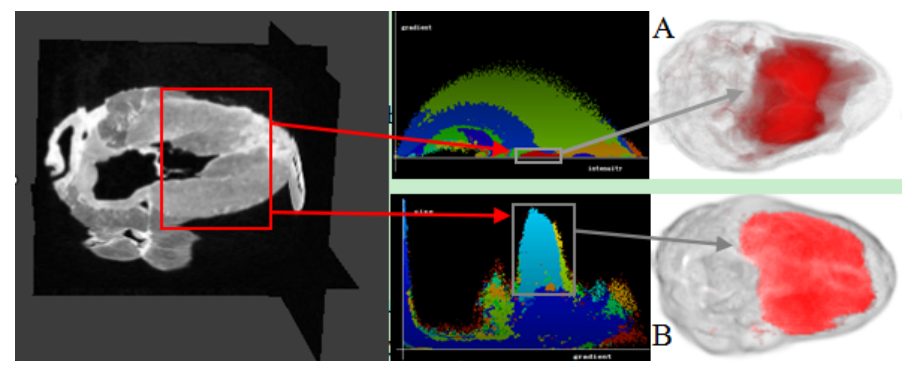

Fig. 5. 3D visualization of sheep heart based on HIG and HIS.
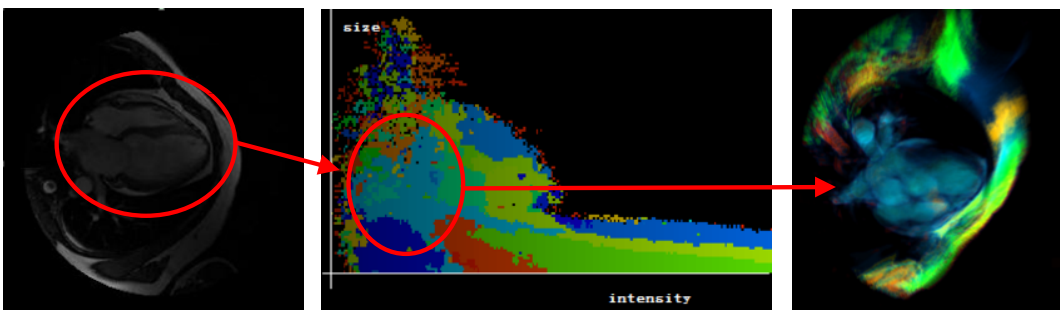

Fig. 6. Visualization of human heart based on hybrid transfer function.

Human cardiac MRI data was also used from [12] for a visualization evaluation, as Figure 6 illustrates. After HIS was generated and classified, the heart voxels were focused on the middle region in the histogram marked by blue. Then, the blue area was selected with the mouse and the parameters to visualize the heart were easily set. Other regions were visualized by HIG using Eq. (3), and the heart was observed easily and clearly.

\section{Conclusion}

Visualization of a heart based on the combination of HIS and HIG was more intuitive than using the HIG histogram alone. By classifying HIS and HIG and assigning different colors to each category, the spatial characteristics and statistical properties can be observed by the user. Therefore, by combining the estimated value for the size information in HIS and the boundary information reflected in HIG, the interactive operability improves.

With the incremental development of technology, high-resolution images are increasingly used, and more effective rendering algorithms are needed. Hence, this work will focus on designing more effective transfer functions for the massive data that will undoubtedly exist in the near future. 


\section{Acknowledgment}

This work was financially supported by National Natural Science Foundation of China (61173086), Shandong Province Science and Technology Development Planning (2012GSF12105) and Shandong Excellent Young Scientist Award Fund (BS2011DX002).

\section{References}

[1] H. Pfister, B. Lorensen, C. Bajaj, G. Kindlmann, W. Schroeder, L.S. Avila, K.M. Raghu, R. Machiraju and J. Lee, The transfer function bake-off, IEEE Compute Graphics and Application 21 (2001), 16-22.

[2] G. Kindlmann and J.W. Durkin, Semi-automatic generation of transfer functions for direct volume rendering, IEEE Symposium on Volume Visualization, 1998, 79-86.

[3] M. Levoy, Display of surface from volume data, IEEE Computer Graphics and Application 8 (1988), $29-37$.

[4] S. Roettger, M. Bauer and M. Stamminger, Spatialized transfer functions, Proceedings of EUROVIS 2005: Eurographics/IEEE VGTC Symposium on Visualization, 2005, 271-278.

[5] R.Z. Huang and K.L. Ma, RGVis: region growing based techniques for volume visualization, Proceedings of the 11th Pacific Conference on Computer Graphics and Applications, 2003, 355-363.

[6] C. Correa and K.L. Ma, Size-based transfer funciton: a new volume exploration technique, IEEE Transaction on Visualization and Computer Graphics 14 (2008), 1380-1387.

[7] C. Correa and K.L. Ma, The occlusion spectrum for volume classification and visualization, IEEE Transaction on Visualization and Computer Graphics 15 (2009), 1465-1472.

[8] M. Kenneth, A survey of visualization pipelines, IEEE Transaction on Visualization and Computer Graphics 19 (2013), 367-378.

[9] F. Yang, W.M. Zuo et al., 3D cardiac MRI data visualization based on volume data preprocessing and transfer function design, Computers in Cardiology 35 (2008), 717-720.

[10] B. Duffy et al., Integrating isosurface statistic and histograms, IEEE Transaction on Visualization and Computer Graphics 19 (2013), 263-277.

[11] G. Madhusudhana, Q.Q. Mohammed, D. Roy et al., Visualization of color anatomy and molecular fluorescence in whole-mouse cryo-imaging, Computerized Medical Imaging and Graphics 35 (2011), 195-205.

[12] C.G. Fonseca, M. Backhaus et al., The Cardiac Atlas Project-An imaging database for computational modeling and statistical atlases of the heart, Bioinformatics 27 (2011), 2288-2295. 